\title{
The quiescent Hubble flow, local dark energy tests, and pairwise velocity dispersion in a $\Omega=1$ universe
}

\author{
P. Teerikorpi ${ }^{1}$, A. D. Chernin ${ }^{1,2,3}$, and Yu. V. Baryshev ${ }^{4,5}$ \\ 1 Tuorla Observatory, University of Turku, 21500 Piikkiö, Finland \\ e-mail: pekkatee@utu.fi \\ 2 Division of Astronomy, University of Oulu, 90014, Finland \\ 3 Sternberg Astronomical Institute, Moscow University, 119899 Moscow, Russia \\ 4 Institute of Astronomy, St. Petersburg State University, Staryj Peterhoff, 198504 St. Petersburg, Russia \\ ${ }^{5}$ Isaac Newton Institute of Chile, Saint-Petersburg Branch, Russia
}

Received 24 March 2005 / Accepted 10 May 2005

\begin{abstract}
We review the increasing evidence for the cosmological relevance of the cold local Hubble flow. New observations, $N$-body simulations and other theoretical arguments are discussed, supporting our previous suggestion that the cosmological vacuum or uniform dark energy can have locally observable consequencies, especially a lower velocity scatter in DE dominated regions. The apparent contradiction between the slight dependence of the growth factor on $\Omega_{\Lambda}$ and the significant influence of dark energy in realistic $N$-body calculations is clarified. An interesting new result is that in the standard $\Lambda$ cosmology, gravitation dominates around a typical matter fluctuation up to about the correlation length $r_{0}$, and we tentatively link this with the high pairwise velocity dispersion on scales up to several Mpc, as measured from galaxy redshift-space correlations. Locally, the smooth Hubble flow on similar scales is consistent with $N$-body simulations including $\Omega_{\Lambda} \approx 0.7$ and a low density contrast in the Local Volume, which make it generally vacuum-dominated beyond 1-2 Mpc from galaxies and groups. We introduce a useful way to view the Hubble flow in terms of "zero gravity" spheres around galaxies: e.g., a set of non-intersecting spheres, observed to be expanding, actually participates in accelerating expansion. The observed insensitiveness of the local velocity dispersion to galaxy mass is explained as an effect of the vacuum, too.
\end{abstract}

Key words. dark matter - cosmological parameters - Local Group

\section{Introduction}

The puzzle of the smooth local Hubble flow was recognized by Sandage et al. (1972) and further emphasized by Sandage (1999) and Thim et al. (2003). From $N$-body simulations Governato et al. (1997) predicted a high velocity dispersion for the Local Group (LG) environment in the case of zero- $\Lambda$ universes. The low velocity scatter was clearly seen in the data analyzed by Ekholm et al. (2001) and Karachentsev \& Makarov (2001). On the theoretical side, Chernin (2001) argued that the smooth vacuum density, starting to dominate over the matter not far from the Local Group (LG), is the dynamical reason for the coldness of the local Hubble flow. We generalized this novel explanation to include the time variable dark energy (DE) density (Baryshev et al. 2001).

In recent years new observations and theoretical studies have only enhanced the importance of the dynamical properties of the Local Group environment. New measurements of distances in the Local Volume have been made. New $N$-body simulations relevant to the local Hubble flow have been performed and a better understanding has been gained on the effect of the cosmological vacuum and $\mathrm{DE}$ on structure formation and peculiar velocities. Redshift-space correlation studies of deep galaxies surveys (2dF, SDSS) have given statistical results on the velocity dispersion on scales comparable to the Local Volume. Here we review these works and discuss the problem further.

\section{New observations of the local Hubble flow}

After our previous studies of the local Hubble flow (Ekholm et al. 2001; Chernin 2001; Baryshev et al. 2001) new observations and analyses have appeared mostly giving support to the picture of a cold flow in the distance range $r \lesssim 5 h_{100}^{-1} \mathrm{Mpc}$, which is also an interesting region in view of the statistical results on the pairwise velocity dispersion from deep galaxy surveys.

\subsection{Individual galaxies}

Davidge \& van den Bergh (2001) measured the distance of the nearby elliptical galaxy Maffei 1 from the asymptotic giant branch tip at near-infrared and obtained the result $\mu=$ $28.2 \pm 0.3$ or $r=4.4(+0.6,-0.5)$ Mpc. With a local Hubble 
constant $\approx 60 \mathrm{~km} \mathrm{~s}^{-1} \mathrm{Mpc}^{-1}$, one predicts a recession velocity of about $264 \mathrm{~km} \mathrm{~s}^{-1}$, which well agrees with the observed $279 \pm 25 \mathrm{~km} \mathrm{~s}^{-1}$.

Gieren et al. (2004) have measured the distance to the nearby (2 Mpc) galaxy NGC 300; It has a previous HST measurement $(\mu=26.50)$, but with over one hundred new Cepheids detected from the ground, the distance could be determined with better accuracy, yielding $\mu=26.43 \pm 0.04(1.93 \mathrm{Mpc})$. This galaxy was listed by Teerikorpi \& Paturel (2002) as having an unbiased Cepheid distance (cf. Sect. 2.3), and the new measurement appears to confirm that. Its radial velocity $112 \mathrm{~km} \mathrm{~s}^{-1}$ well corresponds to the predicted $\approx 116 \mathrm{~km} \mathrm{~s}^{-1}$.

Thim et al. (2003) have measured the Cepheid distance to the spiral galaxy M 83 (NGC 5236) using the Antu $8.2 \mathrm{~m}$ telescope of the ESO VLT. From twelve Cepheids they derived for its dereddened distance modulus the value $28.25 \pm 0.15$, or a distance of $4.5 \pm 0.3 \mathrm{Mpc}$. This distance was consistent with other available distances for the group containing $M$ 83. The mean recession velocity of $249 \pm 42 \mathrm{~km} \mathrm{~s}^{-1}$ is again in agreement with the prediction $\left(\approx 270 \mathrm{~km} \mathrm{~s}^{-1}\right)$ if $h_{100} \approx 0.6$.

Rekola et al. (2004) derived a Cepheid distance to the spiral galaxy IC 342 in the IC 342/Maffei group. Their result, $3.8 \pm$ $0.4 \mathrm{Mpc}$, predicts $V_{\mathrm{c}} \approx 228 \mathrm{~km} \mathrm{~s}^{-1}$, in comparison with the observed $V_{\mathrm{LG}}=230 \mathrm{~km} \mathrm{~s}^{-1}$.

Rekola et al. (2005) measured the distance to NGC 253 by the planetary nebulae luminosity function method and in combination with other methods derived a distance $3.6 \pm 0.2 \mathrm{Mpc}$. This predicts $V_{\mathrm{c}} \approx 216 \mathrm{~km} \mathrm{~s}^{-1}$, in comparison with the measured $V_{\mathrm{LG}}=234 \mathrm{~km} \mathrm{~s}^{-1}$.

\subsection{Karachentsev's TRGB programme}

As one result of the formidable effort to measure distances to as many as possible Local Volume galaxies using the luminosity of the tip of the red giant branch in their programme with the HST and ground telescopes, Karachentsev and his collaborators have confirmed their earlier small values for the velocity dispersion: Karachentsev et al. (2002a,b) using the M 81 group and the Centaurus A group, Karachentsev et al. (2002c) in a study of the very local Huble flow, Karachentsev et al. (2003a) using the Canes Venatici cloud, and Karachentsev et al. (2003b) in a study of local galaxy flows within $5 \mathrm{Mpc}$. Karachentsev et al. (2003c) showed that the centroids of eight nearby galaxy groups have a scatter of about $30 \mathrm{~km} \mathrm{~s}^{-1}$ around the Hubble relation.

\subsection{The Cepheid bias and other studies}

The Cepheid stars, generally regarded as the best primary distance indicators, also contribute to the scatter in the Hubble relation. Teerikorpi \& Paturel (2002) and Paturel \& Teerikorpi (2004) have presented evidence for a selection bias in the Cepheid method, which varies from galaxy-to-galaxy, on average making the measured distances too small. Its influence on the value of the Hubble constant and on the local Hubble diagram has been studied in Paturel \& Teerikorpi (2005).
When one considers the whole sample of Cepheid host galaxies up to the Virgo and Fornax clusters, then the first order corrections to the bias reduce the dispersion around the linear Hubble law from 120 to $84 \mathrm{~km} \mathrm{~s}^{-1}$. When one looks at the nearby volume with $V_{\mathrm{c}}<300 \mathrm{~km} \mathrm{~s}^{-1}$ (or $r \lesssim 5 \mathrm{Mpc}$, then the dispersion descends from $35 \mathrm{~km} \mathrm{~s}^{-1}$ to $31 \mathrm{~km} \mathrm{~s}^{-1}$. It seems that the large scatter in the local Cepheid Hubble diagram, which puzzled Freedman et al. (2001), was partly due to the bias varying from galaxy-to-galaxy.

Whiting (2003) used a sample of local galaxies with distances derived from various methods and sources. He derives a velocity dispersion of about $100 \mathrm{~km} \mathrm{~s}^{-1}$ and suspects that the smaller values derived for example by Ekholm et al. (2001) are due to small number statistics. However, the evidence makes it difficult to reject in this way the reality of a still colder, quite local Hubble flow.

Even a dispersion of around $100 \mathrm{~km} \mathrm{~s}^{-1}$ would be interestingly low cosmologically, as will be discussed below. We regard with great interest the result by Whiting (2003) that the dispersion does not depend on the mass of a galaxy, but is the same for giants and dwarfs. Already noticed by Karachentsev \& Makarov (1996), this phenomenon certainly deserves to be studied with larger and more homogeneous samples.

Macciò et al. (2005) used a sample of 28 galaxies within about $10 \mathrm{Mpc}$ (11 with Cepheid-based distances, 17 early types with SBF distances). From the Hubble diagram they derived the velocity dispersion in spheres of different sizes around the LG and found that $\sigma_{\mathrm{v}}$ varies from $52 \mathrm{~km} \mathrm{~s}^{-1}(r \leq 3 \mathrm{Mpc})$ to $135 \mathrm{~km} \mathrm{~s}^{-1}(r \leq 10 \mathrm{Mpc})$. At small distances, where the distance indicators have the best accuracy, the result is in fair agreement with previous works. At larger distances it may agree with Whiting (2003).

One may ask if the apparent increase of the dispersion towards larger distances, clearly seen in Fig. 1 of Macciò et al. (2005), could be due to some unaccounted-for factors. As the sample extends half-way to the Virgo cluster, some scatter must arise from the systematic differential infall to Virgo, which would shift some of the more distant galaxies upwards in the Hubble diagram. However, such a behaviour is in principle included when one compares observations and realistic simulations. Another effect could be due to the distance indicator. If the derived distance modulus $\mu$ is affected by a Gaussian error with dispersion $\sigma_{\mu}$, the inferred velocity dispersion would increase with distance. In the extreme case, if all dispersion were due to $\sigma_{\mu}$, then the apparent velocity dispersion $\sigma_{\mathrm{v}}$ would increase linearly with the distance, if the Hubble law is linear. We would like to know how strong this effect can be and we give a formula connecting the apparent velocity dispersion $\sigma_{\mathrm{v}}$ with the rms error $\sigma_{\mu}=\sigma$ in the distance modulus at a fixed derived distance $R_{\text {der }}$ (Appendix A):

$\sigma_{\mathrm{v}} \approx H R_{\mathrm{der}}\left[1-\mathrm{e}^{-0.954 \sigma^{2}}\left(2 \mathrm{e}^{-1.70 \sigma^{2}}+\mathrm{e}^{-2.65 \sigma^{2}}\right)\right]^{1 / 2}$.

As a more realistic example, let us assume that $\sigma_{\mu}=0.15$ below $3 \mathrm{Mpc}$ and 0.3 above $6 \mathrm{Mpc}$. Then at small distances the true velocity dispersion would be still about $50 \mathrm{~km} \mathrm{~s}^{-1}$. If it is the same at $10 \mathrm{Mpc}$ (where $V_{\mathrm{c}} \approx 600 \mathrm{~km} \mathrm{~s}^{-1}$ ), then the apparent dispersion would be about $100 \mathrm{~km} \mathrm{~s}^{-1}$. 
Hence we regard still uncertain how large the local increase in $\sigma_{\mathrm{v}}$ with distance actually is. As Macciò et al. (2005) demonstrate, determination of this behaviour provides a local cosmological test when considered together with cosmological $\mathrm{N}$-body simulations, which do predict such a trend in LG-type environments.

\section{The pair-wise velocity dispersion on Mpc-scales from redshift-space correlations}

Recent redshift-space correlation analyses of the SDSS (Hawkins et al. 2003) and 2dF (Zehavi et al. 2002) galaxy surveys have found high pair-wise peculiar velocity dispersions of $500-600 \mathrm{~km} \mathrm{~s}^{-1}$. The method used in those analyses was applied in the classical work by Davis \& Peebles (1983) to the small CfA survey. Such velocity dispersions, together with the cosmic virial theorem, give a value of about 0.3 for the mass density parameter.

Such a large velocity scatter measured within the scale of $\lessgtr 10 h_{100}^{-1}$ Mpc implies that the typical situation in the galaxy universe is that around random galaxies there is practically no detectable Hubble law on such scales. This undoubtly reflects the fact that most galaxies are members of systems from groups to superclusters.

The high pair-wise velocity dispersion on scales of the Local Volume or less is another way to see the problem of the local smooth Hubble flow. In some sense our local environment, where Edwin Hubble was able to find his law, is different from the general picture that emerges from the statistical analysis of redshift-space correlations. This difference could explain the contrast in velocity dispersions.

\section{4. $\Lambda$ and the growth rate of density fluctuation}

Chernin (2001) suggested that a solution to the problem of the cold local Hubble flow may be found in the coincidence that the antigravity of the cosmological vacuum or dark energy starts to dominate over the gravity of lumpy matter at the distance (about $1.5 \mathrm{Mpc}$ ) where the Hubble flow emerges. Thus the relatively low density contrast in the Local Volume allows vast vacuum-dominated regions. This explanation, which we generalized to dark energy (Baryshev et al. 2001), might be criticized on the basis that the influence of the $\Lambda$ term on the growth rate in Friedmann models appears to be miniscule. We discuss this question in this and the next sections.

\subsection{The growth rate and $\Omega_{\wedge}$}

Lahav et al. (1991) derived the following formula for the growth rate at the present epoch $f(z=0) \approx \Omega_{\mathrm{m}}^{0.6}+\frac{1}{70} \Omega_{\Lambda}(1+$ $\left.\frac{1}{2} \Omega_{\mathrm{m}}\right)$. This shows that for a fixed matter density parameter $\Omega_{\mathrm{m}}$, adding the cosmological vacuum into the model has practically no effect for the present growth rate, which also determines peculiar velocities around the growing density fluctuations. In fact, when the vacuum density is added, the growth factor slightly increases.

Lahav et al. (1991) see this insensitivity to $\Lambda$ as reflecting the cosmic vacuum as a uniform background which does not have local force effects. A galaxy does not "feel" the presence of the vacuum. The cosmological constant influences the behaviour of the global scale factor and only in this way enters the differential equations for a growing individual matter density contrast $\delta$.

\subsection{Adding the constraint $\Omega_{m}+\Omega_{\Lambda}=1$}

On the other hand, we have the condition, from the fluctuations of the CBR, that the universe has a flat spatial geometry, so we are constrained to consider the situation $\Omega=\Omega_{\mathrm{m}}+\Omega_{\Lambda}=1$. Then the present growth rate will depend significantly on the fraction of the vacuum $\Omega_{\Lambda}$ in the model. This is clearly seen from the above formula, and in Fig. 1 of Axenides \& Perivolaropuolos (2002) for the growth factor of density fluctuations. The corresponding behaviour of peculiar velocities is seen in Fig. 2 of Peebles (1984), in Fig. 8 of Carroll et al. (1992) and in Fig. 2 of Axenides \& Perivolaropuolos (2002).

For example, compared with the case $\Omega_{\Lambda}=0.0$, peculiar velocities as calculated from the growth rate are a factor of 2 smaller when $\Omega_{\Lambda}=0.7$, while in an extreme case, a factor of 15 smaller when $\Omega_{\Lambda}=0.99$. This does not contradict what was said above, because now changing the vacuum density is accompanied by a change of the matter density parameter.

\section{Dark energy and decay of peculiar velocities}

Another and still more important aspect of the effect of the vacuum follows from the result that in the regions of the universe where dark energy dominates new structures do not condense and linear perturbations of density and peculiar velocities decay (Chernin 2001; Baryshev et al. 2001; Chernin et al. 2003a,b).

This effect was considered for vacuum-dominated regions by Chernin et al. (2003a,b) using the method of stability analysis first suggested by Zeldovich (1965) for Lifshitz-type perturbations in an expanding universe with $\Lambda=0$. It was shown that the inclusion of vacuum can radically change the situation, so that only decreasing or frozen density perturbations are possible in a vacuum-dominated region.

It is important that velocity perturbations, or peculiar velocities, can only decrease in vacuum-dominated regions, where the vacuum acts as an effective cooling agent. In order to see this clearly in a simple situation, we refer the reader to Eqs. (6), (7) in Sect. 7.3. There, Eq. (6) describes the relative velocity of two masses, with the asymptotic $(D \rightarrow \infty)$ velocity $V=H_{\mathrm{V}} D=D / A_{\mathrm{V}}$ corresponding to Hubble's linear velocitydistance relation. Deviations from this regular motion are characterized by a radial peculiar velocity $v$ which is the difference between $\dot{D}$ and $V$. In the simplest case of the parabolic motion, $E=0$, Eq. (6) gives that the peculiar velocity $v$ behaves as:

$v=\dot{D}-D / A_{\mathrm{V}} \propto D^{-2}$.

This result shows that in vacuum-dominated regions the vacuum cooling is even more effective than the usual adiabatic cooling $\left(v \propto a^{-1}\right)$.

The end of new structure formation is an effect of the vacuum. This epoch is usually put at around $z_{\Lambda} \approx 0.7$, when on 
average vacuum starts to dominate over gravitating matter in the homogeneous Friedman universe $\left(1+z_{\Lambda}=\left(2 \Omega_{\Lambda} / \Omega_{\mathrm{m}}\right)^{1 / 3}\right)$. But in the real universe this moment was not the same everywhere, as the matter density varies within a large range. That is why it is useful to adopt the concept of a local "zero-gravity" (ZG) sphere, whose radius can be calculated if one knows the mass distribution and the vacuum or DE density. Locally, beyond such a sphere the vacuum dominates and structure formation has stopped some time ago (the epoch depends on the local matter density).

\section{6. $\mathrm{N}$-body simulations of the local environment as cosmological test}

The fluctuation growth rate analysis of peculiar velocities is concerned with the question of how high velocities are required to maintain a structure growth (the continuity equation tells that one must transfer particles to build up the structure). Such an analysis follows the behaviour of a single growing fluctuation and how the growth is slowed down. We emphasize that it does not consider the cooling of velocities in those vacuumdominated regions where structure formation has stopped. The net effect of all these processes becomes apparent only in realistic $N$-body simulations, such as performed by Klypin et al. (2003) and Macciò et al. (2005). Governato et al. (1997) made important simulations of the local environment within models where $\Omega_{\Lambda}=0$ and arrived at high velocity dispersions, e.g. the flat CDM model gave $300 \mathrm{~km} \mathrm{~s}^{-1}<\sigma_{\mathrm{v}}<700 \mathrm{~km} \mathrm{~s}^{-1}$.

Klypin et al. (2003) simulated the evolution of a region which was similar to our environment within $100 \mathrm{Mpc}$ from the LG. They used the flat $\Lambda \mathrm{CDM}$ model with $\Omega_{\Lambda}=0.3$ and obtained a rather low velocity dispersion of about $60 \mathrm{~km} \mathrm{~s}^{-1}$ in the local Hubble flow.

Macciò et al. (2005) performed $N$-body simulations of flat DE-dominated universes and showed that galaxies around systems similar to the LG have low peculiar velocities. They also showed that replacing the cosmological constant $(w=-1)$ with a DE model with $w=-0.6$, peculiar velocities are still reduced by about 15 percent (their Fig. 4). This is roughly as expected on the basis of our simple analytic calculation in Sect. 5.4 in Baryshev et al. (2001), where it was argued that the longer adiabatic cooling in the quintessence model with $w=-2 / 3$ leads to lower peculiar velocities. One may suppose that a still lower velocity dispersion might have been found for our third example, the coherently evolving model with $w=-2 / 3$, where the cooling time is still longer. This model is of special interest as it produces a Hubble relation that is close to the standard $\Lambda$-model relation fitting the SNIa observations for $z \lesssim 1.5$ (Teerikorpi et al. 2004).

Macciò et al. (2005) concluded that two facts are essential for one to reproduce the low local velocity dispersion: 1) a correct cosmology, requiring the $\Lambda$ or DE component; and 2) a correct environmental density contrast, such as around the LG, which appears to be rather small (0.2-0.6). If the density contrast is large, then high peculiar velocities are expected even in DE cosmologies. This is qualitatively understandable because then the ZG surface extends to a greater distance and the present gravity-dominated region is larger.
We note that in the Macciò et al. simulations the mean value of the local Hubble constant within a few Mpc is close to the global one $\left(70 \mathrm{~km} \mathrm{~s}^{-1} \mathrm{Mpc}^{-1}\right)$, with a majority of individual simulated "Local Volumes" having values between 60 and 80 (Macciò, private communication).

A different kind of local Hubble flow calculation was made by Chernin et al. (2004). They traced the trajectories of local galaxies back to the epoch of the formation of the LG and found initial conditions that were very different from those that would directly lead to the linear Hubble flow. With simulations they identified the vacuum as the agent that introduces the subsequent regularity in the nearby flow.

We conclude that the concepts of gravity- and vacuumdomination as well as the size of gravity-dominated region are useful tools when one studies structure formation and the evolution of peculiar velocities.

\section{Characteristics of the zero-gravity surface}

The dynamics of a spherically symmetric dust matter cloud with density $\rho_{\mathrm{m}}(r)$ on the homogeneous DE background is described by the Einstein's field equations (Chernin 2001; Baryshev et al. 2001), giving the following exact equation of motion:

$\ddot{r}=-G M_{\mathrm{eff}} / r^{2} ; \quad M_{\mathrm{eff}}=M_{\mathrm{m}}(r)+M_{\mathrm{DE}}(r)$.

Here $M_{\mathrm{m}}(r)=4 \pi \int_{0}^{r} \rho_{\mathrm{m}}(r) r^{2} \mathrm{~d} r$ is the dust mass within the sphere of radius $r, M_{\mathrm{DE}}(r)$ is the DE mass within the same radius, given as $M_{\mathrm{DE}}=(4 \pi / 3)(1+3 w) \rho_{\mathrm{DE}} r^{3}$.

\subsection{The zero-gravity radius}

For the point-mass model there is a distance $r_{\mathrm{ZG}}$ where $\ddot{r}=0$ and the DE gravitating mass equals that of the matter cloud, i.e. $M_{\text {eff }}=0$. For the cosmological constant $(w=-1)$ this "zerogravity radius" is

$r_{\mathrm{ZG}}=\left(3 M /\left(8 \pi \rho_{\Lambda}\right)\right)^{1 / 3}$.

For a point mass $r_{\mathrm{ZG}}$ remains always constant. In the standard flat universe with $\Omega_{\Lambda}=0.7$ a mass $M_{\mathrm{LG}}=210^{12} M_{\odot}$ has $r_{\mathrm{ZG}}=1.5 h_{60}^{-2 / 3} \Omega_{\Lambda}^{-1 / 3} \mathrm{Mpc}$. Chernin et al. (2004) calculated the ZG surface around the Local Group, dominated by the Milky Way and M 31 pair, and found that it is almost spherical and remains nearly unchanged during a 12.5 Gyr history of the LG.

The ZG sphere for a point mass $M$ has special significance in an expanding universe. A light test particle at $r>r_{\mathrm{ZG}}$ experiences an acceleration outwards. If it has even a small recession velocity away from $M$, it participates in an accelerated expansion.

One may also define another interesting sphere with an "equal energy" radius $r_{\mathrm{EE}}$. In such a sphere around the mass $M$ the matter and vacuum energies are equal. Its radius is somewhat larger than the $\mathrm{ZG}$ radius: $r_{\mathrm{EE}}=2^{1 / 3} r_{\mathrm{ZG}} \approx 1.26 r_{\mathrm{ZG}}$. This radius means for two identical point masses the same as the $\mathrm{ZG}$ radius for a test particle. Separated by the distance $D=r_{\mathrm{EE}}$ the two masses have zero acceleration relative to the centre-ofmass, while for $D>r_{\mathrm{EE}}$ they experience outward acceleration. 
These examples illustrate in simple situations the general result that in vacuum-dominated expanding regions, perturbations do not grow (Sect. 5).

\subsection{The Hubble flow of $Z G$ spheres}

In physics four kinds of mass appear: active gravitational mass, defined above as $M_{\text {eff }}$, passive gravitational mass $M_{\text {pas }}$, inertial mass $M_{\text {ine }}$, and the mass responsible for the gravitational potential (in Newtonian terms) $M_{\text {pot }}$. These masses are equivalent for zero-pressure non-relativistic matter. However, for vacuum the masses per unit volume are $\rho_{\mathrm{V}}+3 P_{\mathrm{V}}=-2 \rho_{\mathrm{V}}, \rho_{\mathrm{V}}+P_{\mathrm{V}}=0$, $\rho_{\mathrm{V}}+P_{\mathrm{V}}=0$, and $\rho_{\mathrm{V}}$, respectively. The equivalence principle tells us that passive and inertial masses are equivalent; they are both zero for vacuum that does not feel any gravity and is not affected by matter.

It is interesting and useful to formulate the Hubble flow in terms of ZG (or EE) spheres. A typical galaxy together with its massive halo is well contained within such a cell $\left(r_{\mathrm{ZG}} \approx 1\right.$ Mpc for $5 \times 10^{11} M_{\odot}$ ) as are groups of the LG type, which are scattered in the Local Volume ${ }^{1}$. Viewed in this way, cosmological expansion is the relative motion of the set of ZG spheres. To a first approximation, the space between them contains just vacuum.

A ZG sphere has zero effective gravitating mass, but nonzero passive (and inertial) gravity mass, so it feels the global gravity field. The equation that describes (in the centre-of-mass frame) the relative motion of two ZG spheres (neglecting first all other ones) has the mathematical structure of Friedmann's cosmological equation:

$\ddot{D}=D / A_{\mathrm{V}}^{2}\left[1-\left(r_{1}^{3}+r_{2}^{3}\right) / D^{3}\right]$,

where $D$ is the distance between the centres of the cells with (constant) ZG radii $r_{1}$ and $r_{2}$, and $A_{\mathrm{V}}=\left(\frac{8 \pi G}{3} \rho_{\mathrm{V}}\right)^{-1 / 2}$. Note that for two equal masses $\left(r_{1}=r_{2}=r_{\mathrm{ZG}}\right)$, the acceleration is positive, if $D>2^{1 / 3} r_{\mathrm{ZG}}=r_{\mathrm{EE}}$, as was already pointed out above. In general $\ddot{D}>0$ if $D>\left(r_{1}^{3}+r_{2}^{3}\right)^{1 / 3}$. For $r_{1} \geq r_{2}$, this is always valid if $D>2^{1 / 3} r_{1}$, which means that the mass point 2 lies outside of the EE sphere of the mass 1 .

If we add other mass points sparsely enough so that the above condition is fulfilled (i.e. each EE sphere contains only its own point mass), the net accelerations relative to the centreof-mass remain positive and if the spheres are originally at rest relative to each other or are recessing, the system will scatter with accelerating expansion.

We may consider Eq. (5) for a spherical distribution (the radius $=D$ of non-intersecting $Z G$ spheres with radia $r_{\mathrm{ZG}}$ and a light test particle on its surface. Taking the equivalent $\mathrm{ZG}$ sphere in the centre, it has $\left.R_{\mathrm{ZG}}=D r_{\mathrm{ZG}}[(4 \pi / 3) n)^{1 / 3}\right]$ where $n$ is the number density of the $\mathrm{ZG}$ spheres. Then one derives for the test particle $\ddot{D}=D / A_{\mathrm{V}}^{2}\left[1-(4 \pi / 3) n r_{\mathrm{ZG}}^{3}\right]>0$, which reduces to the familiar Friedmann equation containing the mean matter density $\rho_{\mathrm{m}}$ and the vacuum density $\rho_{\mathrm{V}}$.

A useful implication is the following: if we see a region where the galaxies and groups are so sparse that their EE

\footnotetext{
${ }^{1}$ However, for a large cluster or a supercluster, the ZG spheres contain a progressively smaller fraction of the volume.
}

spheres do not contain other objects and if this region is expanding, one may conclude that there is accelerating expansion approaching the global Hubble expansion rate and no further structure formation.

\subsection{Towards the insensitivity to galaxy mass}

Considering further the case of two spheres, the first integral of the equation of motion is

$\dot{D}^{2}=\left(D / A_{V}\right)^{2}\left[1+2\left(r_{1}^{3}+r_{2}^{3}\right) / D^{3}\right]+2 E$.

For parabolic expansion $(E=0)$, the local Hubble "constant" squared becomes

$H^{2}=\frac{8 \pi G}{3} \rho_{V}\left[1+2\left(r_{1}^{3}+r_{2}^{3}\right) / D^{3}\right]$.

If one forgets other ZG spheres, the Hubble expansion rate for these two galaxies is close to the value $H_{\mathrm{V}}=1 / A_{\mathrm{V}}$ depending on the vacuum density only. Because $(r / D)^{3}$ decreases quickly with increasing considered distance $D$, such a situation may occur in sparsely populated regions. Looking at large regions containing many galaxies, the Friedmann equation and the Hubble expansion rate depend on the vacuum and mean matter densities (the end of Sect. 7.2). In the standard cosmology, $\left(1+\rho_{\mathrm{m}} / \rho_{\Lambda}\right)^{1 / 2}=1.195$, meaning that the "full" global Hubble constant is 20 percent larger than the rate due to vacuum only. For example, if $H_{0}=72 \mathrm{~km} \mathrm{~s}^{-1} \mathrm{Mpc}^{-1}$, $H_{\mathrm{V}}=60 \mathrm{~km} \mathrm{~s}^{-1} \mathrm{Mpc}^{-1}$.

Equation (7) gives some insight to why the Hubble law is equally well followed by massive and light galaxies as noted in Sect. 2.3 (Karachentsev \& Makarov 1996; Whiting 2003). For a massive halo of $4 \times 10^{12} M_{\odot}$ the distance $r_{i}$ is about $1.9 \mathrm{Mpc}$, hence the factor $2\left(r_{1} / D\right)^{3}$ is quite small already at $D=5 \mathrm{Mpc}$ and still smaller for smaller haloes. For both massive and light haloes the square root of the bracketed expression in Eq. (7) differs little from unity on a range of spatial scales. Thus in this simplified model one measures both for large and small galaxies practically the same Hubble constant that basically depends on the dominating vacuum density.

Viewing the Local Volume as a sparse set of ZG spheres, on wide areas between the groups it is vacuum-dominated (Karachentsev et al. 2003c) and all galaxies follow the accelerating expansion not far from the global Hubble rate. Whiting (2003) listed possible explanations for the mass independence in the local dynamics. His explanation No. 2 would need very massive dark objects that similarly affect different galaxies. In our explanation the massive dark vacuum is the agent, but in the sense that it causes accelerated expansion with its rate varying in a narrow range and which is automatically accompanied by vacuum cooling, as we explained in Sect. 5.

\subsection{Vacuum and the bulk motion}

Although there is a rather regular Hubble flow around us in the Local Volume, this same volume has a bulk motion relative to the cosmic background raditation. The motion is similar to that of the LG, or about $630 \mathrm{~km} \mathrm{~s}^{-1}$ (Karachentsev et al. 2003c, 
give a summary of bulk motion measurements). How is it possible for a regular Hubble expansion to exist on a scale $\sim H_{0} R$ superposed on a stream with $V \sim H_{0} R$ ? Chernin (2001) and Chernin et al. (2003b) pointed out that the bulk motion and the Hubble flow may co-exist, because vacuum is co-moving with any motion: two frames of reference may move with respect to each other with any velocity, but the vacuum looks identical to them and has the same effects. If it regularizes the Hubble flow in a vacuum-dominated region which is at rest relative to the CBR, it does the same for a region moving as a whole relative to the CBR. Thus the vacuum appears to be relevant for two aspects of the local Hubble flow: its regularity and identity with the global flow, and its insensitivity to the simultaneous large-scale motion.

\section{Typical scales of gravity-dominated regions}

It is possible to calculate typical scales for gravity-dominated regions at the present epoch, assuming that light traces mass and using the results of galaxy correlation analysis.

\subsection{The $Z G$ radius and the correlation length}

In the classical two-point correlation function analysis, one assumes that one may present the average fluctuation of the mass density around a given galaxy as

$\rho(r)=\bar{\rho}_{\mathrm{m}}\left[1+\left(r / r_{0}\right)^{-\gamma}\right]$

where $\bar{\rho}_{\mathrm{m}}$ is the average mass density, $r_{0}$ is the so-called correlation length, and $\gamma$ is the correlation exponent. Integrating over the distance $r$ from 0 to $r$ one obtains a typical mass $M_{\mathrm{m}}(r)$ within this scale:

$M_{\mathrm{m}}(r)=\frac{4 \pi}{3} r^{3} \bar{\rho}_{\mathrm{m}}\left[1+\frac{3}{3-\gamma}\left(r / r_{0}\right)^{-\gamma}\right]$.

The vacuum with its constant energy density $\rho_{\Lambda}$ starts dominating relative to this fluctuation at and beyond the distance where $M_{\Lambda}(r) \geq M_{\mathrm{m}}(r)$, meaning that the radius of the ZG sphere $r_{\mathrm{ZG}}$ is obtained from

$\left[1+\frac{3}{3-\gamma}\left(r_{\mathrm{ZG}} / r_{0}\right)^{-\gamma}\right]=2 \frac{\rho_{\Lambda}}{\rho_{\mathrm{m}}^{-}}$.

With the values generally regarded as standard, i.e. $r_{0} \approx$ $5 h_{100}^{-1} \mathrm{Mpc}$ and $\gamma \approx 1.75$ for the correlation function, and $\Omega_{\Lambda}=0.7, \Omega_{\mathrm{m}}=0.3$, one calculates

$r_{\mathrm{ZG}}=0.8 r_{0} \approx 4 h_{100}^{-1} \mathrm{Mpc}$.

\subsection{Scales of high pair-wise peculiar velocities}

So we see that typically the gravity-dominated region in the standard $\Lambda$ universe has a radius not far from the observed correlation length. The corresponding scale is about $2 r_{0}$, which is also the region where the recent redshift-space correlation analyses of the SDSS (Hawkins et al. 2003) and 2dF (Zehavi et al. 2002) galaxy surveys have found high pair-wise peculiar velocity dispersions, $500-600 \mathrm{~km} \mathrm{~s}^{-1}$. We make a few comments in terms of gravity- and vacuum-domination:

1) As such galaxies are now in a gravity-dominated region, they were also in the past. This is because in a spherically symmetric fluctuation (without shell crossings) a galaxy at $r_{\mathrm{ZG}}$ marks this distance all the time (the mass inside the radius defined by the galaxy remains constant, hence $r_{\mathrm{ZG}}$ is constant for a constant vacuum).

2) The gravity-dominated region within $\approx r_{0}$ presents a matter density contrast of $2 \rho_{\Lambda} / \rho_{\mathrm{m}}-1 \approx 3.7$. According to Fig. 2 in Macciò et al. (2005), one would then expect in a Local Volume sized sphere a velocity dispersion of $300-400 \mathrm{~km} \mathrm{~s}^{-1}$, corresponding to a pair-wise dispersion of about $500 \mathrm{~km} \mathrm{~s}^{-1}$, as observed in SDSS and $2 \mathrm{dF}$.

3) There are indications both in SDSS and $2 \mathrm{dF}$ that the pair-wise velocity dispersion drops beyond about $2 r_{0}$, i.e. there where the deprojected mutual distances are generally larger than the $\mathrm{ZG}$ distance. It is tempting to suggest that we are here seeing the same effect as in the Local Volume, where the smooth Hubble flow starts immediately after the directly calculated zero-gravity surface.

4) Beyond $10 h_{100}^{-1} \mathrm{Mpc}$ the measured velocity dispersion is $\ll H R$ implying a Hubble flow. As we are now in the vacuumdominated region, there is accelerating expansion and no further structure formation.

\section{Conclusions}

We summarize our conclusions:

- Recent observations on the Local Volume are consistent with a low velocity dispersion around the local Hubble flow and almost the same local and global Hubble constants.

- New cosmological $N$-body simulations by Klypin et al. (2003) and Macciò et al.(2005) give support to our hypothesis that the inclusion of the cosmological vacuum or smooth dark energy produces lower velocity dispersions in vacuum-dominated regions.

- The concept of vacuum domination is a useful tool for characterizing different regions in the galaxy distribution. It is helpful in general to view a region of the galaxy universe in terms of "zero-gravity" or "equal-energy" spheres surrounding galaxies and groups.

- Within the standard picture of a two-point correlation function for galaxy distribution, the vacuum-domination generally starts around the correlation length $r_{0}$ in the standard flat $\Lambda$ universe.

- Recent statistical results from the SDSS and 2dF surveys on the amplitude and scale-dependence of pair-wise velocity dispersion are consistent both with our view of the importance of vacuum-domination and with the low velocity dispersion in the Local Volume.

- Locally, we have the advantage that the border of zerogravity can be directly derived from known local masses. The smooth Hubble flow observed beyond this distance is consistent with the $N$-body simulations including $\Lambda \approx 0.7$ and a low local density contrast. 
- The similar local Hubble flow for both massive and light galaxies may naturally reflect the dynamical dominance of the vacuum.

Acknowledgements. This study has been supported by The Academy of Finland (project "Fundamental questions of observational cosmology") and by the foundation Turun Yliopistosäätiö. We thank the referee for useful comments and A. Macciò for communicating unpublished results on $N$-body simulations.

\section{Appendix A: Velocity scatter and distance errors}

Let us assume that a distance indicator has a Gaussian distribution of the accidental error in the derived distance modulus, with the dispersion $\sigma_{\mu}=\sigma$. An error $\delta$ in the modulus, when the true distance is $R$ and the Hubble law is valid, may be interpreted as a peculiar velocity $v_{\text {pec }}$ at a constant cosmological velocity $V$ :

$v_{\text {pec }}=H_{0} R\left(1-10^{0.2 \delta}\right)$.

As the error $\delta$ has a Gaussian distribution, the apparent dispersion squared around the Hubble law is obtained as $\left(H_{0} R\right)^{2}\langle(1-$ $\left.\left.10^{0.2 \delta}\right)^{2}\right\rangle$, or:

$\sigma_{\mathrm{v}}^{2}=\left(H_{0} R\right)^{2} \frac{1}{\sqrt{2 \pi} \sigma_{\mu}} \int_{-\infty}^{\infty}\left(1-2 \mathrm{e}^{a \delta}+\mathrm{e}^{2 a \delta}\right) \mathrm{e}^{-\delta^{2} / 2 \sigma^{2}} \mathrm{~d} \delta$

where $a=0.2 \ln 10=0.46$.

However, as we do not know the true cosmological velocity, it is better to consider the apparent velocity dispersion at a fixed derived distance. Then one must write for the peculiar velocity:

$v_{\mathrm{pec}}=H R_{\mathrm{der}}\left(10^{-0.2 \delta}-1\right)$.

When now calculating the average, one must weight the usual Gaussian error distribution with the distribution $f\left(\mu_{\text {app }}-\delta\right)=$ $f(\mu)$, giving the relative number of true distance moduli feeding the subsample having the fixed derived distance modulus $\mu_{\mathrm{der}} \pm$ $\frac{1}{2} \mathrm{~d} \mu_{\mathrm{der}}$. For the case of a radial density distribution $\propto R^{-\alpha}$ and a magnitude-limited sample: $f\left(\mu_{\text {app }}-\delta\right) \propto \mathrm{e}^{k \mu}$, where $k=0.2(3-$ $\alpha) \ln 10$, the integration results in

$\sigma_{\mathrm{v}}=H R_{\operatorname{der}}\left[1-\mathrm{e}^{-\frac{k^{2}}{2} \sigma^{2}}\left(2 \mathrm{e}^{-\frac{(k+a)^{2}}{2} \sigma^{2}}+\mathrm{e}^{-\frac{(k+2 a)^{2}}{2} \sigma^{2}}\right)\right]^{1 / 2}$.

E.g., for a homogeneous distribution $(\alpha=0)$ :

$\sigma_{\mathrm{v}} \approx H R_{\operatorname{der}}\left[1-\mathrm{e}^{-0.954 \sigma^{2}}\left(2 \mathrm{e}^{-1.70 \sigma^{2}}+\mathrm{e}^{-2.65 \sigma^{2}}\right)\right]^{1 / 2}$.

\section{References}

Axenides, M., \& Perivolaropoulos, L. 2002, Phys. Rev. D, 65, 7301A Baryshev, Yu., Chernin, A., \& Teerikorpi, P. 2001, A\&A, 378, 729

Carroll, S., Press, W., \& Turner, E. 1992, ARA\&A, 30, 499

Chernin, A. 2001, Physics-Uspekhi, 44, 1099

Chernin, A. D., Karachentsev, I. D., Valtonen, M. J., et al. 2004, A\&A, 415,19

Chernin, A. D., Nagirner, D. I., \& Starikova, S. V. 2003a, A\&A, 399, 19

Chernin, A., Teerikorpi, P., \& Baryshev, Yu. 2003b, Adv. Space Res., 31,459

Davidge, T. J., \& van den Bergh, S. 2001, ApJ, 553, L133

Davis, M., \& Peebles, P. J. E. 1983, ApJ, 267, 465

Ekholm, T., Baryshev, Yu., Teerikorpi, P., Hanski, M., \& Paturel, G. 2001, A\&A, 368, L17

Freedman, W. L., Madore, B., Gibson, B. K., et al. 2001, ApJ, 553, 47

Gieren, W., Pietrzyński, G., \& Walker, A. 2004, AJ, 128, 1167

Governato, F., Moore, B., Cen, R., et al. 1997, New Astr., 2, 91

Hawkins, E., Maddox, S., Cole, S., et al. 2003, MNRAS, 346, 78

Karachentsev, I., \& Makarov, D. 1996, AJ, 111, 794

Karachentsev, I., \& Makarov, D. 2001, Astrofiz., 44, 5

Karachentsev, I. D., Dolphin, A. E., Geisler, D., et al. 2002a, A\&A, 383,125

Karachentsev, I. D., Sharina, M. E., Dolphin, A. E., et al. 2002b, A\&A, 385,21

Karachentsev, I. D., Sharina, M. E., Makarov, D. I., et al. 2002c, A\&A, 389,812

Karachentsev, I. D., Sharina, M. E., Dolphin, A. E., et al. 2003a, A\&A, 398, 479

Karachentsev, I. D., Makarov, D. I., Sharina, M. E., et al. 2003b, A\&A, 398,479

Karachentsev, I. D., Chernin, A. D., \& Teerikorpi, P. 2003c, Astrofiz., 46,491

Klypin, A., Hoffman, Y., Kravtsov, A., \& Gottloeber, S. 2003, ApJ, 596

Lahav, O., Lilje, P., Primack, J., \& Rees, M. 1991, MNRAS, 251, 126

Macciò, A. V., Governato, F., \& Horellou, C. 2005, MNRAS, 359, 941

Paturel, G., \& Teerikorpi, P. 2004, A\&A, 413, L31

Paturel, G., \& Teerikorpi, P. 2005, A\&A, in press

Peebles, P. J. E. 1984, ApJ, 284, 439

Rekola, R., Flynn, C., McCall, M.L., et al. 2004, in R. Rekola: Distance Determinations to Nearby Galaxies, Turku University Ph.D. Thesis, Annales Universitatis Turkuensis, Ser. AI t., 329

Rekola, R., Richer, M. G., McCall, M. L., et al. 2005, MNRAS, in press

Sandage, A. 1999, ApJ, 527, 479

Sandage, A., Tammann, G., \& Hardy, E. 1972, ApJ, 172, 253

Teerikorpi, P., Gromov, A., \& Baryshev, Yu. 2004, A\&A, 407, L9

Teerikorpi, P., \& Paturel, G. 2002, A\&A, 381, L37

Thim, F., Tammann, G., Saha, A., et al. 2003, ApJ, 590, 256

Whiting, A. B. 2003, ApJ, 587, 186

Zehavi, I., Blanton, M. R., Frieman, J. A., et al. 2002, ApJ, 571, 172

Zeldovich, Ya. B. 1965, Advan. Astron. Ap., 3, 241 